\title{
On the Use of Conformal Models and Methods in Dosimetry for Nonuniform Field Exposure
}

DOI:

10.1109/TEMC.2017.2723459

\section{Document Version}

Accepted author manuscript

Link to publication record in Manchester Research Explorer

\section{Citation for published version (APA):}

Poljak, D., Cvetkovic, M., Bottauscio, O., Hirata, A., Laakso, I., Neufeld, E., Reboux, S., Warren, C., Giannopolous, A., \& Costen, F. (2017). On the Use of Conformal Models and Methods in Dosimetry for Nonuniform Field

Exposure. IEEE Transactions on Electromagnetic Compatibility, 60(2), 1-10.

https://doi.org/10.1109/TEMC.2017.2723459

Published in:

IEEE Transactions on Electromagnetic Compatibility

\section{Citing this paper}

Please note that where the full-text provided on Manchester Research Explorer is the Author Accepted Manuscript or Proof version this may differ from the final Published version. If citing, it is advised that you check and use the publisher's definitive version.

\section{General rights}

Copyright and moral rights for the publications made accessible in the Research Explorer are retained by the authors and/or other copyright owners and it is a condition of accessing publications that users recognise and abide by the legal requirements associated with these rights.

\section{Takedown policy}

If you believe that this document breaches copyright please refer to the University of Manchester's Takedown Procedures [http://man.ac.uk/04Y6Bo] or contact uml.scholarlycommunications@manchester.ac.uk providing relevant details, so we can investigate your claim.

\section{OPEN ACCESS}




\title{
On the Use of Conformal Models and Methods in Dosimetry for Nonuniform Field Exposure
}

\author{
Dragan Poljak, Senior Member, IEEE, Mario Cvetković, Member, IEEE, Oriano Bottauscio, Senior Member, IEEE, \\ Akimasa Hirata, Fellow, IEEE, Ilkka Laakso, Member, IEEE, Esra Neufeld, Sylvain Reboux, Craig Warren, \\ Antonis Giannopolous, and Fumie Costen, Senior Member, IEEE
}

\begin{abstract}
Numerical artifacts affect the reliability of computational dosimetry of human exposure to low-frequency electromagnetic fields. In the guidelines of the International Commission of Non-Ionizing Radiation Protection, a reduction factor of 3 was considered to take into account numerical uncertainties when determining the limit values for human exposure. However, the rationale for this value is unsure. The IEEE International Committee on Electromagnetic Safety has published a research agenda to resolve numerical uncertainties in low-frequency dosimetry. For this purpose, intercomparison of results computed using different methods by different research groups is important. In previous intercomparison studies for low-frequency exposures, only a few computational methods were used, and the computational scenario was limited to a uniform magnetic field exposure. This study presents an application of various numerical techniques used: different finite-element method (FEM) schemes, method of moments, and boundary-element method (BEM) variants, and, finally, by using a hybrid FEM/BEM approach. As a computational example, the induced electric field in the brain by the coil used in transcranial magnetic stimulation is investigated. Intercomparison of the computational results is presented qualitatively. Some remarks are given for the effectiveness and limitations of application of the various computational methods.
\end{abstract}

Manuscript received February 21, 2017; revised June 13, 2017; accepted June 25, 2017. (Corresponding author: Akimasa Hirata.)

D. Poljak and M. Cvetković are with the Faculty of Electrical Engineering, Mechanical Engineering and Naval Architecture, University of Split, 21000 Split, Croatia (e-mail: dpoljak@fesb.hr; mcvetkov@fesb.hr).

O. Bottauscio is with the Istituto Nazionale di Ricerca Metrologica, 10135 Turin, Italy (e-mail: o.bottauscio@inrim.it).

A. Hirata is with the Department of Computer Science and Engineering, Nagoya Institute of Technology, Nagoya 466-8555, Japan (e-mail: ahirata@ nitech.ac.jp).

I. Laakso is with the Department of Electrical Engineering and Automation, Aalto University, FI-00076 Espoo, Finland (e-mail: ilkka.laakso@ aalto.fi).

E. Neufeld is with the IT'IS Foundation for Research on Information Technologies in Society, CH-8092 Zürich, Switzerland (e-mail: neufeld@itis. ethz.ch).

S. Reboux is with the ZMT Zurich MedTech, 8004 Zürich Switzerland (e-mail: reboux@zurichmedtech.com).

C. Warren is with the Department of Mechanical and Construction Engineering, Northumbria University, Newcastle upon Tyne NE1 8ST, U.K. (e-mail: craig.warren@northumbria.ac.uk).

A. Giannopoulos is with the Institute of Infrastructure and Environment, School of Engineering, University of Edinburgh, Edinburgh EH9 3JL, U.K. (e-mail: a.giannopoulos@ed.ac.uk).

F. Costen is with the School of Electrical and Electronic Engineering, The University of Manchester, Manchester M13 9PL, U.K. (e-mail: fumie.costen@ manchester.ac.uk).

Color versions of one or more of the figures in this paper are available online at http://ieeexplore.ieee.org.

Digital Object Identifier 10.1109/TEMC.2017.2723459
Index Terms-Induced fields, low-frequency dosimetry, simplified brain model, sphere brain model, transcranial magnetic stimulation (TMS).

\section{INTRODUCTION}

$\mathbf{H}$ UMAN exposure to artificial electromagnetic fields has raised an increasing public concern regarding adverse health effects [1]. The assessment of low-frequency (LF) exposure is based on the evaluation of internal current density [2] or internal electric field [3], [4], while high-frequency (HF) exposure is based on the evaluation of specific absorption rate averaged over 1 or $10 \mathrm{~g}$ of tissue, which is a surrogate of temperature rise.

In addition to environmental exposure to man-made electromagnetic fields due to steadily increasing number of power and telecommunication installations, efficient medical treatments and diagnosis using electromagnetic radiation also require the knowledge of the accurate distribution of the electromagnetic fields inside the tissues. As it is rather difficult, or even impossible, to measure directly these quantities, the use of computational methods becomes necessary to determine internal field distributions [5]-[10].

There exists two international guidelines/standard for LF exposure mentioned by the World Health Organization. In the IEEE C95.6 standard [4], the ellipsoid is considered to derive the external and internal field strength. The International Commission of Non-Ionizing Radiation Protection (ICNIRP) guidelines [3] use computational results using anatomical models. Although the developed high-resolution anatomically based models provide the detailed body representation currently available for LF dosimetry, there are some aspects that may need consideration. In 2014, the IEEE International Committee on Electromagnetic Safety (ICES) Technical Committee 95 Subcommittee 6 (EMF Dosimetry Modeling) has been established to resolve uncertainties and advance proper use of numerical models to determine electric fields induced within the body due to external electromagnetic fields or contact currents [11].

Voxel models suffer from essential errors due to stair-casing approximations, especially when discretized at another resolution than the underlying voxels. ICNIRP, thus, considers a reduction factor of 3 to account for numerical uncertainty. Historically, the difference of the induced electric field in anatomical models was suggested to be large in the intercomparison by 
Stuchly and Gandhi [12]. In that study, different human body models with different sets of electrical conductivities were used, together with a discussion on sensitivity of the electrical conductivities. Hirata et al. [13] coordinated the intercomparison using the same anatomical model named TARO with an identical set of electrical conductivities. The 99th percentile value of the induced electric field in the human body models, which is recommended in the ICNIRP guidelines [3] as the dosimetric quantity, is in good agreement for uniform magnetic field exposures. However, it is difficult to be certain of the reliability of the 99th percentile value of the induced electric field because no exact analytic solution exists for a realistic anatomical model. It is particularly applicable to nonuniform magnetic exposure as first suggested in [14]. Additional issue is how to process the internal electric field averaged over 2-mm cube [3], as discussed in [15].

The suitability of the applied numerical solution methods is then related to the highly heterogeneous electrical properties of the body and the complexity of the external and internal geometry. The numerical methods for LF exposure scenarios range from simple canonical models, e.g., [16], [17], robust finite difference scheme, e.g., [18], [19], which are ideally suited for simulations of high-resolution inhomogeneous models, but limited to scenarios where the wavelength is not too big compared to the resolution, to the approaches suitable for adaptive conformal meshes, such as finite-element method (FEM), e.g., [20], [21] or boundary-element method (BEM), e.g., [22], [23]. It should be noted that the numerical method is not necessarily fixing the discretization approach. For example, while the FEM frequently uses adaptive unstructured meshes, many FEM implementations (including some of those used in this work) employ structured, rectilinear meshes, or voxels. Conversely, variants of the finite-difference time-domain (FDTD) method support subcell models, conformal corrections, or local adaptivity.

Recent advancements in LF dosimetry have been reported by a number of researchers, e.g., Chan et al. [24], De Santis et al. [25], Hirata et al. [26], Dimbylow and Findlay [27], Laakso et al. [28], Neufeld et al. [29], Kuster [15], [30], and others, in addition to sensitivity analysis [6], [22], [31]. In most of the studies, uniform field exposure was considered. As summarized above, no study conducts intercomparison for LF nonuniform exposure. Dosimetry for nonuniform field becomes essential for product safety and medical applications.

This study summarizes comparison on the implementation of conformal models in LF dosimetry. For benchmarking purposes, different research groups have carried out calculations for nonuniform exposure. Unlike previous intercomparisons at low frequencies, several computational methods were implemented. As an example, the electric field induced in the brain by transcranial magnetic stimulation (TMS) coil is considered.

\section{METHODS AND MODELS}

\section{A. Stair-Cased and Conformal Methods}

Contrary to simple canonical models used in early dosimetry papers (plane slab, cylinders, homogeneous and layered spheres, and prolate spheroids), modern realistic anatomically based computational models comprising of cubical cells are mostly related to the use of the FDTD method [10], scalar-potential finite difference method, or the FEM applied to structured meshes. The conformal FEM, BEM, method of moments (MoM), and some other methods are, on the other hand, being used to a somewhat lesser extent.

Undoubtedly, an advantage of conformal methods, such as $\mathrm{BEM}$, is that such methods themselves represent the natural way of avoiding stair-casing error in terms of the implementation of curvilinear or isoparametric elements. Furthermore, for different alternatives (e.g., quasi-static solvers and integral equation method) to full-wave methods, there is no need to implement absorbing boundary conditions. Using integral equation methods and some MoM approaches, one typically avoids volume meshes and reduce number of elements for large-scale problems, at the cost of difficult handling of inhomogeneity. On the other hand, serious drawbacks of integral equation methods, such as BEM, are more complex formulation (particularly for nonhomogeneous domains) and corresponding numerical implementation. Namely, numerical implementation of integral methods leads to dense matrices being computationally far more expensive than FDM and FEM. Also, the problem of Green function singularities/quasi-singularities has to be solved within any integral equation scheme. A recent study [7] presents a short review of the use of some integral methods in LF and HF dosimetry, respectively.

\section{B. Exposure Scenario and Model}

The implementation of several numerical methods such as FDTD, FEM, BEM, FEM/BEM, and MoM, respectively, to the LF dosimetry problem has been investigated on the TMS setup, for the simple geometry of sphere and a more realistic, but still simplified, geometry of the brain.

While human exposure to fields generated by different electromagnetic sources has raised a number of questions regarding potential adverse health effects, some biomedical applications of electromagnetic fields in medical diagnostic and for therapy purposes, such as TMS, recently become of particular importance [10], [32], [33], as evidenced by modeling efforts of several investigators [9], [10], [14], [34].

As an initial exploration to the subject, it has been proposed to compare the results using a homogenized realistic-shaped brain reported in [10]. Having verified the model compatibility with the FEM and the MoM, it has been decided that the TMS setup is well suited for initial comparisons.

Hence, a simple sphere homogeneous brain model and an homogenized realistic-shaped brain model have been prepared by using several discretization schemes (from coarse to fine), as shown in Table I.

The models have been initially prepared for MATLAB use. The script for the viewing purposes has been prepared, as well. The initial sphere of $1 \mathrm{~m}$ radius has been scaled using a factor of 0.06 , corresponding to $12 \mathrm{~cm}$ diameter. Dimensions of the brain model are width $13.18 \mathrm{~cm}$, length $16.11 \mathrm{~cm}$, and height $13.9 \mathrm{~cm}$. 
TABLE I

SPECIFIED GEOMETRY PARAMETERS

\begin{tabular}{lccc}
\hline \hline Geometry & Points & Triangles & Tetrahedra \\
\hline sphere_199_244_809 & 199 & 244 & 809 \\
Sphere_406_494_1690 & 406 & 494 & 1690 \\
sphere_803_734_3815 & 803 & 734 & 3815 \\
brain_250 & 232 & 360 & 814 \\
brain_500 & 483 & 696 & 1871 \\
brain_800 & 885 & 1224 & 3542 \\
brain_1200 & 1405 & 1870 & 5771 \\
\hline \hline
\end{tabular}

Furthermore, frequency-dependent parameters of the homogeneous models are taken from [10], i.e., relative permittivity and electric conductivity, respectively, are $\varepsilon=46940$, $\sigma=0.0859 \mathrm{~S} / \mathrm{m}$, at $f=2.44 \mathrm{kHz}$. In addition, linear and isotropic behavior is assumed for the electrical properties of tissues.

For the TMS coils, three generic geometries have been considered, namely, standard circular coil, Figure- 8 coil, and butterfly coil (Figure- 8 with wings inclined $10^{\circ}$ ). The coil operating frequency is $2.44 \mathrm{kHz}$, while the radius, impressed current, and number of turns are given in [10]. Each coil is located $1 \mathrm{~cm}$ over the surface of the model. The exact location of coil center (circular, 8-coil) is determined from the location of the model nodes: $V_{X}=\operatorname{mean}(\operatorname{node}(:, 1))+$ $C_{x} ; \quad V_{Y}=\operatorname{mean}(\operatorname{node}(:, 2))+C_{y} ; \quad V_{Z}=\max (\operatorname{node}(:, 3))+$ $C_{z}$, where $\left.C_{x}=C_{y}=0, C_{z}=0.01\right)$ are displacement of coil center ( $1 \mathrm{~cm}$ over primary motor cortex). From this geometric center, location of all other coil elements is determined.

\section{Numerical Methods Implemented in Comparison}

The following numerical methods have been used in the TMS setup comparison: surface integral equation (SIE)-based MoM (SIE/MoM) carried out by Cvetković et al. [7], [10]; the FEM with cubical elements carried out by Laakso and Hirata [14], the BEM and the hybrid FEM/BEM carried out by Bottauscio et al. [35], [36], and the FEM with rectilinear elements using Sim4Life software carried out by Neufeld and Reboux. Interested readers can found specifics on the particular formulation type and the related solution method in the above references.

At low frequencies, the electric and magnetic fields are decoupled, and it is possible to treat the exposure to these fields separately. Another property of LF exposures is that for most of the tissues, the conduction currents are at least one order of magnitude higher than the displacement currents, and therefore, in most of the scenarios, only tissue conductivity is considered, while the permittivity can be neglected.

\section{NUMERICAL RESULTS}

\section{A. Fundamental Discussion}

First, the intercomparison of various methods with the analytical solution is carried out for the case of a homogeneous sphere exposed to infinitesimal magnetic dipole. The goal was to evaluate the induced field strength around the surface for localized or nonuniform exposure. A dielectric sphere of radius $8 \mathrm{~cm}$ centered at the origin is exposed to a magnetic dipole source located $3 \mathrm{~cm}$ above the sphere with dipole moment oriented in the $z$-direction.

The analytical approach to analyze a multilayer sphere with arbitrary isotropic material parameters ( $\varepsilon$ and $\sigma$ ) exposed to magnetic and/or electric dipoles based on Mie theory provides a full-wave solution and works at any frequency (previously confirmed at both $50 \mathrm{~Hz}$ and in the gigahertz range [37]).

The set of points is selected along three lines $(x-, y$-, and $z$-axes) and two surfaces $(2 \mathrm{~mm}$ and $2 \mathrm{~cm}$ below the sphere surface), respectively. Fig. 1 shows the electric and magnetic fields along three axes for the analytical case, numerical solution obtained using quasi-static FEM with cubical elements, and full-wave SIE/MoM solution. As evident from Fig. 1, analytical and numerical results computed by FEM are in excellent agreement, while SIE/MoM results do not match satisfactorily. These discrepancies particularly occur for points located close to the sphere surface where rather sharp peaks are observed and are thus a serious drawback for the integral-equation-based solution due to a strong singularity of the kernel. Further SIE/MoM calculations using various mesh resolutions also showed a more pronounced effect near the surface. Hence, the results by current implementation of SIE/MoM at this LF scenario should not be taken without scrutiny. It is a well-known fact that the electric field integral equation, on which SIE/MoM is based, suffers from an LF breakdown problem [38]. In order to avoid this and improve the results, it is necessary to use the so-called loop-tree decomposition of basis functions followed by a frequency normalization of the matrix system. More details could be found in [38].

Fig. 2 shows comparisons between the induced electric field over two spherical surfaces obtained analytically and numerically by the FEM. Again, a very good agreement is shown, while the largest difference between the FEM and the analytical solution seems to be at points with peak field values.

\section{B. Some Specifics Related to Implemented Hybrid FEM/BEM}

The set of results, obtained using the BEM code with triangular surface elements and the FEM/BEM code featuring voxel elements, are based on two different formulations: the complete formulation (where E and B fields are both unknowns) and the approximate one (where only $\mathrm{E}$ is a problem unknown). The latter is valid if the reaction of the induced currents on the magnetic field can be disregarded.

The solutions with the FEM/BEM voxel model are obtained by creating a voxel model of the object (sphere or brain) having approximately the same number of volume tetrahedral elements reported in Table I. As voxel models are a structured mesh (used for highly anatomical human models), the resolution can be low in some regions, without loss of shape adaptivity, but with insufficient resolution of the field inhomogeneity. This fact can explain some discrepancies with BEM results. Simulations assuming voxels of smaller size ( 1 and $2 \mathrm{~mm}$ ) have been also carried out.

\section{Some Specifics Related to Simulation Setup for the FEM With Rectilinear Elements (Sim4Life) Study}

Regarding the implementation of models in the FEM code, the brain geometries were imported as .stl (standard tessellation 

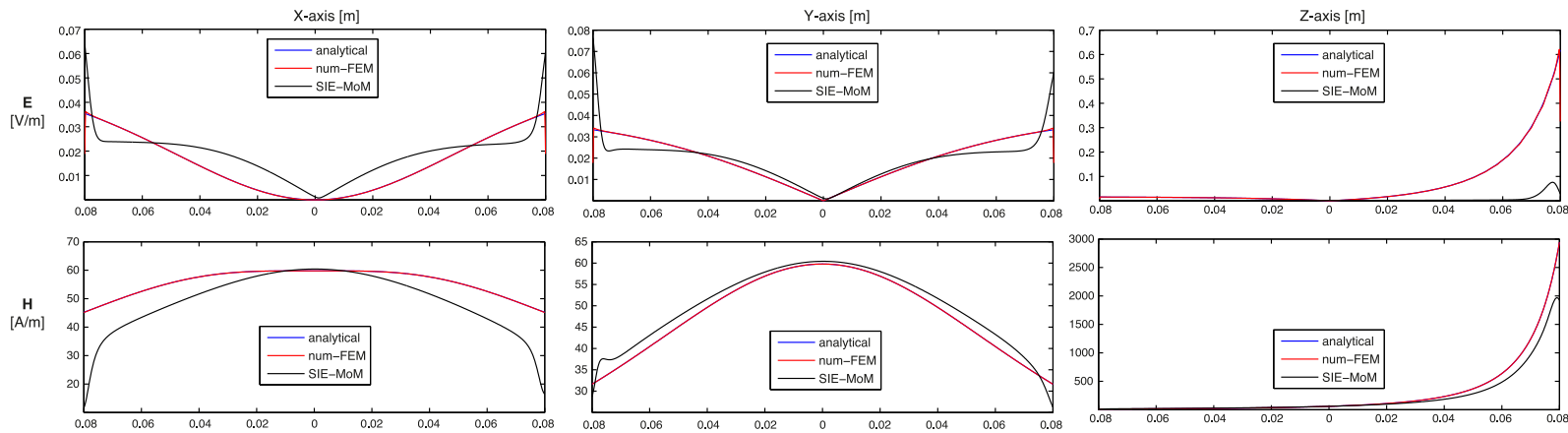

Fig. 1. Distribution of electric field (top) and magnetic field (bottom) along three axes. Comparison between analytical solution (analytical), FEM with cubical elements (num-FEM), and SIE/MoM (SIE-MoM).
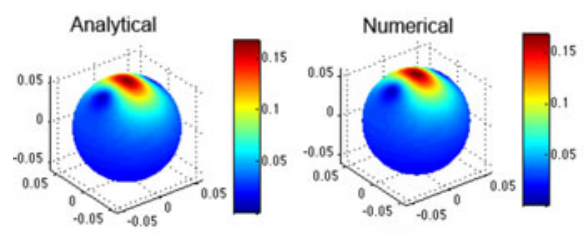

(a)
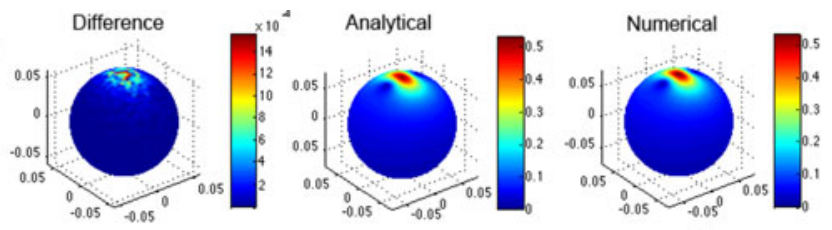

(b)

Fig. 2. Maps of induced electric field over a spherical surface (a) $2 \mathrm{~cm}$ below and (b) $2 \mathrm{~mm}$ below, respectively. Comparison between analytical solution and numerical solution using the FEM with cubical elements (side length of $0.5 \mathrm{~mm}$ ).

TABLE II

CRITERIA OF RELEVANCE TO THE SUITABILITY OF USING A MAGNETO-QUASI-STATIC APPROXIMATION

\begin{tabular}{lcc}
\hline \hline & Sphere & Homogeneous brain \\
\hline Criteria $\sigma / \omega \varepsilon$ & 13.5 & 13.5 \\
Criteria $\omega^{2} \varepsilon \mu d^{2}$ & $\sim 1.7 \cdot 10^{-5}$ & $\sim 2.3 \cdot 10^{-5}$ \\
Criteria $\omega \sigma \mu d^{2}$ & $\sim 0.00023$ & $\sim 0.0003$ \\
\hline \hline
\end{tabular}

language) format in Sim4Life. The sphere is created directly using the embedded CAD modeling tool. Its triangulated surface was edited such that no edge is larger than $4 \mathrm{~mm}$.

The coil models do not account for the separation between the coil windings, so all the turns collapse into a single wire. The current source is thus modeled with one turn. The current intensity (peak amplitude, not RMS) is determined from [10] as the number of turns times the coil current. For both the 8-Coil and Butterfly Coil, the currents in the two coil parts flow in opposite directions.

As evidenced from Table II, for both the sphere and the realistic brain model, the ratio $\sigma / \omega \varepsilon$ is 13.5 , which is significantly larger than 1, thus indicating that the ohmic-current-dominated flavor of the magneto-quasi-static equation can be used.

The tolerance for the relative residuals of the magneto-quasistatic (convergence criterion) solver was set to $10^{-12}$. Selected results show peak amplitude distributions of the induced electric fields and magnetic flux densities.

\section{Spherical Model}

The first set of results is related to a TMS coil positioned $1 \mathrm{~cm}$ over a homogeneous spherical model. The comparison is given for the induced electric field and magnetic flux density maps, respectively, on a cross section of the sphere model, as shown in Fig. 3. The results have been obtained using SIE/MoM, FEM with cubical elements, complete and approximate BEM, complete and approximate FEM/BEM, and FEM codes, respectively. Also, Table III gives a comparison of maximum induced electric field (V/m) and magnetic flux density (T) obtained using different numerical models for the case of circular coil and spherical geometry.

The results for the induced electric field obtained using different methods agree relatively satisfactorily as evidenced from the cross-sectional maps. Results for the magnetic flux density in the same cross-sectional plane are a plausible match, as well. Still, there are some numerical artifacts evident in the results using SIE/MoM code, which could be attributed to the low number of field points in the interpolation scheme.

\section{E. Simplified Brain Geometry}

The following comparison between the same numerical methods has been performed on a simplified brain model. Fig. 4 shows the distribution of the induced electric field at the brain surface due to three typical TMS coils: circular, figure-of-8, and butterfly, obtained using SIE/MoM and FEM. On the other hand, Fig. 5 shows distribution of the induced electric field on the coronal cross section of the brain model. The results have been obtained using SIE/MoM, FEM with cubical elements, complete and approximate BEM, complete and approximate FEM/BEM, and FEM codes, respectively. Table IV gives a comparison of maximum induced electric field $(\mathrm{V} / \mathrm{m})$ obtained using different numerical models for the case of circular coil.

The comparison from Fig. 5 demonstrates that the results computed using the quasi-static solver and the FEM method and the full wave analysis carried out via SIE/MoM, 

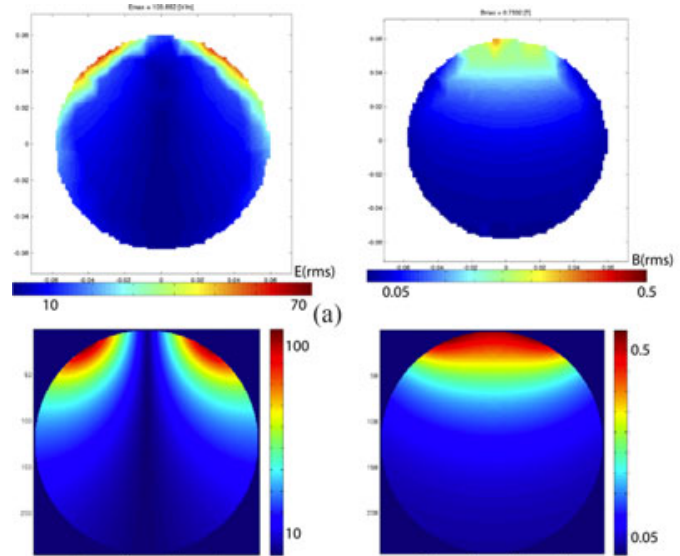

(b)

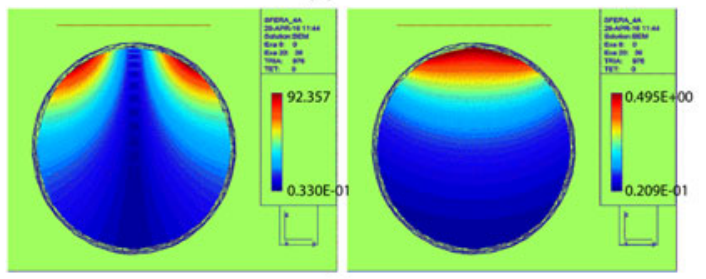

(c)

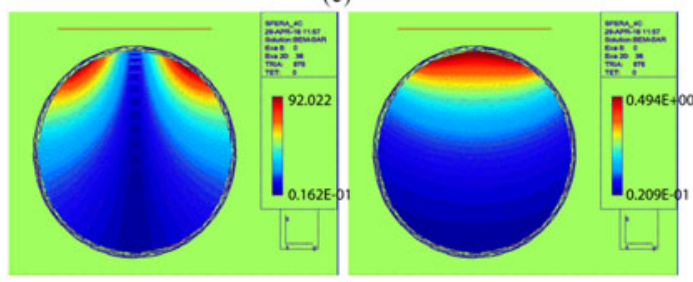

(d)

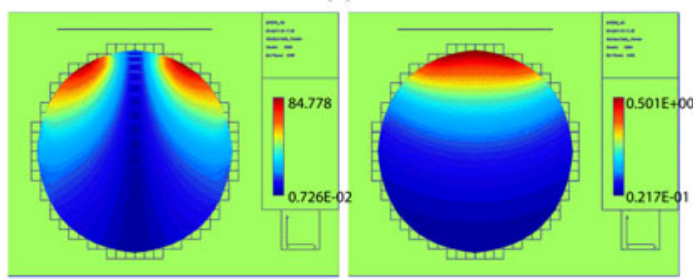

(e)

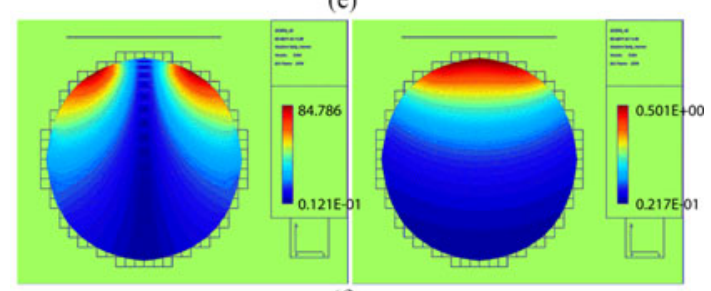

(f)
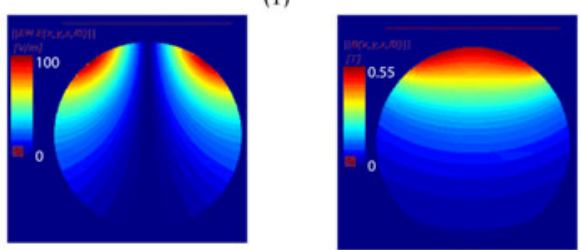

(g)

Fig. 3. Maps of the induced electric field (left column) and the magnetic flux density (right column) for conformal and stair-cased spherical geometry, respectively, due to circular coil. Results obtained by (a) SIE/MoM with 976 triangles, (b) FEM with cubical elements with a side length of $0.5 \mathrm{~mm}$, (c) approximate BEM, and (d) complete BEM using 976 triangles, respectively, (e) approximate FEM/BEM, (f) complete FEM/BEM using 5394 voxel elements $(5.5 \mathrm{~mm})$, respectively, and (g) FEM using grid resolution of $0.5 \mathrm{~mm}$. respectively, do not exactly match. The electric field distribution over the cross section is similar, but the maximum values obtained by different methods differ somewhat. Finally, some initial investigation related to the numerical errors due to discretization and convergence was done using the structured mesh FEM code (voxels) by varying the Cartesian grid step, the triangulated mesh density of the brain surface, and the residual tolerance of the iterative solver. Results are shown in Fig. 6 for the strictest criteria and finest resolutions for the geometry of the brain.

A comparison of results computed for different grid resolutions indicates good convergence for the field values inside the brain (or the sphere). A thorough convergence analysis has not yet been performed. The maximum field values are located on the surface of the objects and thus converge more slowly.

\section{DISCUSSION}

The intercomparison of numerical results by involved research groups obtained a reasonable agreement in the induced electric fields.

Comparison with an analytical solution for a sphere exposed to dipole field showed excellent agreement with results obtained using the FEM with cubical elements, while SIE/MoM results do not match satisfactorily. Additional SIE/MoM calculations using various mesh resolutions showed this effect to be more pronounced near model surface.

The results for a sphere and a simplified brain model exposed to circular and butterfly coils obtained using SIE/MoM, BEM, and FEM codes, respectively, agree relatively satisfactorily as evidenced from the cross-sectional maps of the induced electric field. Results for the magnetic flux density in the same cross-sectional plane are a plausible match, as well. Still, there are some numerical artifacts evident in the results using the SIE/MoM code. The electric field distribution over the cross section is similar, but the maximum values obtained by different methods differ somewhat.

Initial investigation using the FEM code related to the numerical errors due to different grid resolutions showed good convergence for the field values inside the brain and the sphere.

When comparing different numerical results in the literature, the observed differences can be related to factors such as human model size and detailedness, posture, organ size and shape, dielectric properties, the exposure source model, boundary conditions, and numerical factors (accuracy of the numerical method, discretization resolution, mesh quality, and convergence). While all of these are important factors when talking about comparing different studies, most of these should not be relevant for intercomparisons with clearly defined setups as all should use the same geometry, same properties, etc. In the presented intercomparison, the observed differences are partly related to the different numerical techniques, but unfortunately also to insufficiently well-defined setup specificationthe exact source positioning (angles) relative to the brain model and the evaluation planes and points were not specified, making quantitative comparison impossible. Hence, it is suggested that in future work, much of the differences resulting from 
TABLE III

Comparison of Maximum Induced Electric Field (V/M) And MAgnetic Flux Density (T) ObTained Using DifFerent Numerical Models For the CASE of Circular CoIl AND SPHERICAL GeOMETRY

\begin{tabular}{|c|c|c|c|c|c|c|c|c|}
\hline \multicolumn{4}{|c|}{ Surface, conforming } & \multicolumn{3}{|c|}{ Voxels, nonconforming } & \multicolumn{2}{|c|}{ Surface, conforming } \\
\hline & Triangles & BEM complete & BEM approximate & Voxels & FEM/BEM complete & FEM/BEM approximate & Triangles & SIE \\
\hline \multirow{3}{*}{$\mathrm{E}[\mathrm{V} / \mathrm{m}]$} & 494 & 90,0 & 89,6 & $1718(8 \mathrm{~mm})$ & 70,1 & 70,1 & 494 & 105,6 \\
\hline & 734 & 90,5 & 91,7 & $4105(6 \mathrm{~mm})$ & 92,2 & 92,2 & 734 & 105,7 \\
\hline & 976 & 92,0 & 92,4 & $5347(5,5 \mathrm{~mm})$ & 84,8 & 84,8 & 976 & 108,3 \\
\hline \multirow[t]{2}{*}{$\mathrm{B}[\mathrm{T}]$} & 244 & 0,505 & 0,504 & $854(10 \mathrm{~mm})$ & 0,501 & 0,501 & 244 & 0,581 \\
\hline & 976 & 0,494 & 0,495 & $5347(5,5 \mathrm{~mm})$ & 0,501 & 0,501 & 976 & 0,561 \\
\hline
\end{tabular}

BEM computations (BEM complete/approximate), hybrid FEM/BEM computations (FEM/BEM complete/approximate), and SIE-MoM computations, respectively.
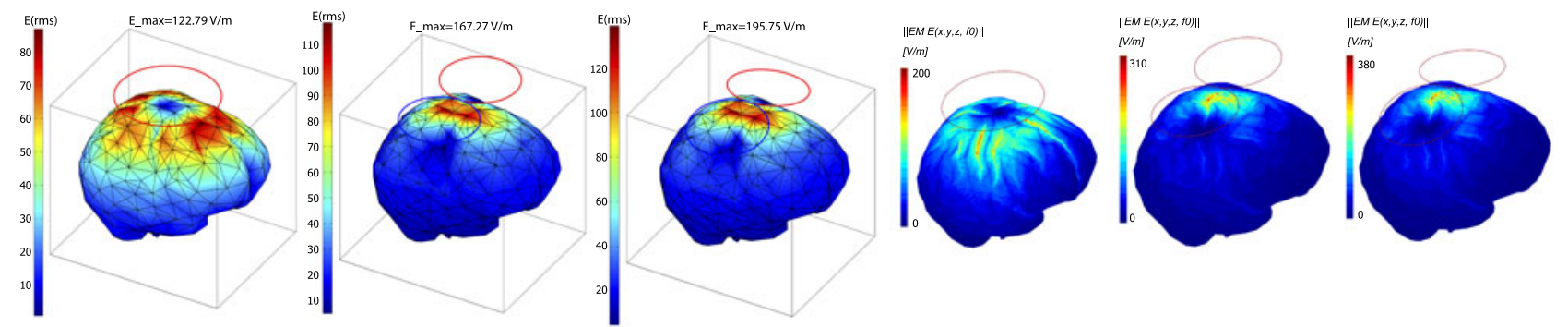

Fig. 4. Maps of the induced electric field over the brain surface. Top to bottom: circular coil, Figure- 8 coil, and butterfly coil. Results obtained by SIE/MoM for conformal surface comprising of 1224 triangles (left) and FEM (right) using grid resolution of $0.5 \mathrm{~mm}$.

comparison at different planes and setups are to be overcome by giving specific set of evaluation trajectories and also including precise source information, in order to allow more quantitative comparison the type of approximation introduced by the different methods.

When comparing the methods, their performance (including scaling with increasing resolution), accuracy, and strengths (e.g., ability of dealing with inhomogeneity) must be investigated, while considering the interdependence of these factors. For example, methods based on voxels are more likely to introduce stair-casing artifacts, but cannot directly be compared with methods featuring a similar number of conformal elements, as the structuredness of their discretization facilitates scaling to higher resolutions, thus reducing discretization errors. In general, a good comparison should require the methods to first perform a convergence study to determine the method-specific requirements to get a converged solution. Then, the solution quality and computational efforts can be compared.

Surface plots should be interpreted and compared carefully, due to the combination of interpolation to the surface, field discontinuity at interfaces, and surface element orientation discontinuities at edges. The cross section and line plots are more reliable to get quantitative information.

\section{A. Limitations}

This study featured comparison on only very simple problems (homogeneous, isotropic, single, mostly smooth surface, without internal structure or inclusions), although some of the employed approaches can perform calculation on very detailed geometries at high resolution (already within this study, in the case of the FEM method using cubical elements, the finest spherical mesh has been discretized using $0.5-\mathrm{mm}$ cubical elements, equivalent to 7.4 million degrees of freedom). On the other hand, calculations using the SIE/MoM code could be undertaken only on a coarsely discretized geometry. In addition, using the SIE/MoM, the field values at intermediate points are calculated using an interpolation scheme. Low number of elements and field determined at restricted number of points will result in some numerical artifacts particularly evident on the crosssectional results for the magnetic flux density. This could be overcome by determining the field at higher resolution.

As previously highlighted, the employed conformal brain geometry is still an extremely simplified model (mainly for the purpose of comparison). The surface is radically smoothed (missing folding structures of gyri and sulci) and the model consists of a single homogeneous structure. Although it is more realistic than the sphere, it lacks the detailed cortical structures and inhomogeneity (gray/white matter, ventricles, etc.). In order to overcome this limitation, the future work should, therefore, include comparisons on the detailed anatomically correct head model, featuring complex material maps and shapes.

Nonetheless, regarding the use of the homogeneous model, it is important to emphasize that it is reasonable to start comparing different numerical techniques using simple models, thus opening the subject. In any case, one has to deal with discrepancies in numerical results due to complexity of the geometry and material (inhomogeneity). It was shown in some previous 


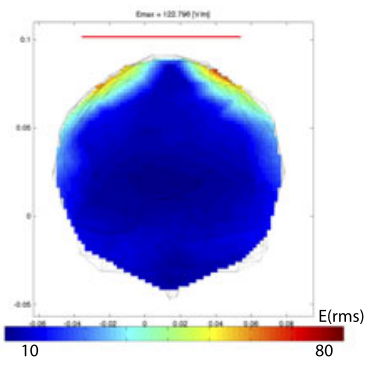

(a)

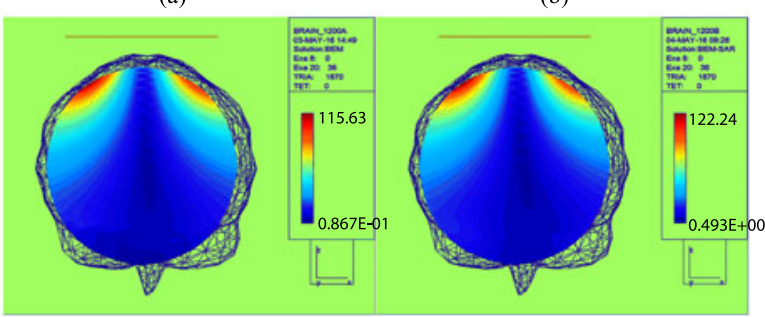

(c)

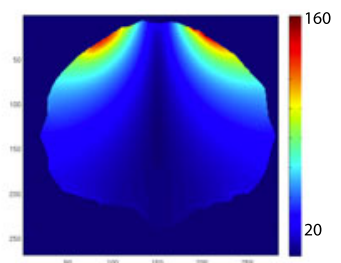

(b)

(d)

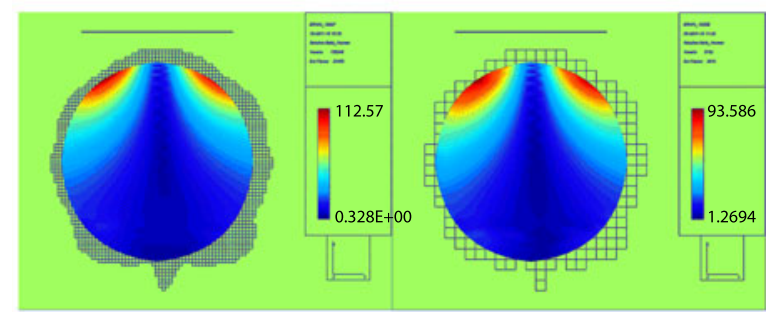

(e)

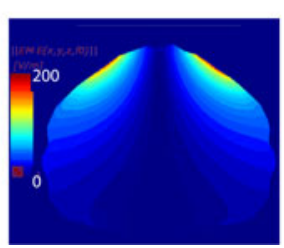

(g)

Fig. 5. Maps of the induced electric field for conformal and stair-cased brain geometry, respectively, due to circular coil. Results obtained by: (a) SIE/MoM with 976, (b) FEM with cubical elements, (c) approximate BEM, and (d) complete BEM using 1870 triangles, respectively, (e) approximate FEM/BEM using 155546 voxels $(2 \mathrm{~mm}$ ), (f) complete FEM/BEM using 5394 voxel elements $(5.5 \mathrm{~mm})$, and $(\mathrm{g})$ FEM using grid resolution of $0.5 \mathrm{~mm}$.

papers [39] that computational artifacts are caused at the airtissue boundary. It was also shown in some previous papers that integral equation techniques are more sensitive to irregularities in geometry than inhomogenities, e.g., [6], [22].

Also, since there is only a qualitative comparison between the methods, any future effort should include the quantitative comparison between the different methods (either in terms of accuracy or in terms of computational effort) as well as detailed convergence study.

\section{CONCLUDING REMARKS}

There are several aspects to be improved in the human dosimetry for LF field exposure, as suggested in the Research Agenda by the IEEE ICES [11]. One of the specific issues is the stair-casing error arising from the commonly used voxel anatomic models, which could be removed by using conformal methods, such as BEM or FEM.

The present paper reviewed and presented intercomparison on the use of various numerical techniques applied to
TABLE IV

COMPARISON OF MAXIMUM INDUCED ELECTRIC FIELD USING VARIOUS Numerical Models For the CASE OF BRAin GEOMETRY AND CiRCUlaR TMS COIL

\begin{tabular}{lccc}
\hline \hline Triangles & SIE & Triangles & BEM, complete \\
\hline 360 & 115,9 & 696 & 117,1 \\
696 & 122,8 & 1870 & 122,2 \\
1224 & 134,5 & Voxels & FEM/BEM, approx. \\
Triangles & BEM, approx. & $5762(6 \mathrm{~mm})$ & 93,6 \\
696 & 108,6 & & FEM/BEM, complete \\
1870 & 115,6 & $5762(6 \mathrm{~mm})$ & 93,6 \\
\hline \hline
\end{tabular}

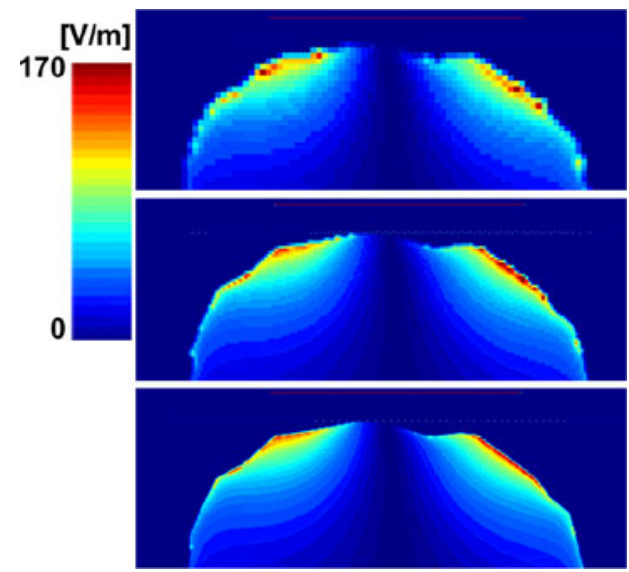

Fig. 6. Induced electric field (peak) at different grid resolutions for the circular coil, at the centered sagittal cross section. Top to bottom: $2 \mathrm{~mm}, 1 \mathrm{~mm}$, and $0.5 \mathrm{~mm}$. Results obtained using structured mesh FEM (voxels).

conformal models in LF dosimetry. Unlike previous intercomparisons [12], [13], nonuniform exposure was considered and several computational methods were used. We particularly discussed the differences attributable to the implementation of methods for nonuniform exposure. The implementation of MoM, FEM, BEM, and hybrid FEM/BEM has been investigated on the TMS setup, for the geometry of a sphere and of a conformal simplified geometry of a homogeneous isotropic brain. Illustrative computational examples related to the assessment of the induced field in the brain are given in the paper.

\section{APPENDIX}

\section{ON THE APPLICATIONS OF FDTD AT VERY LOW FREQUENCIES}

The suitability of applying the FDTD technique to dosimetry at very low frequencies has been examined by E. Neufeld from ETH Zurich, Switzerland, C. Warren and A. Giannopoulos from the University of Edinburgh, U.K., and F. Costen from Manchester University, U.K., with regard to the reference TMS setups.

Researchers from the University of Edinburgh have access to a full body model (AustinMan-http://bit.ly/AustinMan) [40], which can be used in their FDTD simulation software (gprMax-http://www.gprmax.com) [41]. The brain gray and white matter of the brain have been extracted from the full body model, which is meshed with $2 \times 2 \times 2 \mathrm{~mm}$ cells. As an initial step, a magnetic dipole can be used to simulate the coil.

However, the main problem in using the FDTD method for this scenario remains the low excitation frequency. Using a 
2-mm spatial resolution coupled with a $2.44-\mathrm{kHz}$ excitation, results in an unfeasibly large number of iterations, and hence simulation time, e.g., for 1-ms duration simulation, $\sim 260 \times 10^{6}$ iterations ( 2.5 months with moderate parallelization, although this is very implementation dependent) are required. Therefore, without moving to higher excitation frequencies, it is not really feasible to run a FDTD simulation.

As far as the FDTD simulations are concerned, for LF dosimetry, it can be stated that at such low frequencies (kilohertz frequency range with geometry in $10^{-1} \mathrm{~m}$ range), FDTD is not suitable. This is because the maximal stable time step relative to the EM time period is proportional to the ratio of grid step to wavelength. The spatial discretization required for FDTD is usually around $1 / 10$ of the wavelength at the frequency of interest, with an additional need to resolve the skin depth, while in LF simulations at very low frequencies, the resolution required to resolve the geometry is much finer than that which results in an extremely large number of time steps for a simulation. Even when applying various established numerical techniques or algorithms, such as a wide variety of implicit schemes, subgridding and subcell methods, frequency scaling, or innovative source models, FDTD simulations would require unfeasibly long durations. For example, a simulation of the model setup with a time window of $300 \mu$ s for $\Delta x=1 \mathrm{~cm}$, which is the very coarse sampling of the object, would need to run more than $10^{7}$ time steps.

\section{REFERENCES}

[1] D. Poljak, "Electromagnetic fields: Environmental exposure," in Encyclopedia of Environmental Health. Amsterdam, The Netherlands: Amsterdam, The NetherlandsElsevier, 2011.

[2] International Commission on Non-Ionizing Radiation Protection (ICNIRP), "Guidelines for limiting exposure to time-varying electric, magnetic and electromagnetic fields (up to $300 \mathrm{GHz}$ )," Health Phys., vol. 74, no. 4, pp. 494-522, 1998.

[3] International Commission on Non-Ionizing Radiation Protection (ICNIRP), "Guidelines for limiting exposure to time-varying electric and magnetic fields (1 Hz to $100 \mathrm{kHz}$ )," Health Phys., vol. 99, no. 6, pp. 818-836, 2010.

[4] IEEE Standard for Safety Levels With Respect to Human Exposure to Electromagnetic Fields, O-3 kHz, IEEE Std C95.6-2002, Oct. 2002.

[5] J. Hand, "Modelling the interaction of electromagnetic fields (10 MHz-10 $\mathrm{GHz}$ ) with the human body: Methods and applications," Phys. Med. Biol., vol. 53, no. 16, pp. R243-R286, 2008.

[6] D. Poljak, D. Cavka, H. Dodig, C. Peratta, and A. Peratta, "On the use of the boundary element analysis in bioelectromagnetics," Eng. Anal. Boundary Elements, vol. 49, pp. 2-14, 2014.

[7] D. Poljak, M. Cvetković, A. Peratta, C. Peratta, H. Dodig, and A. Hirata, "On some integral approaches in electromagnetic Dosim," in Proc. Joint Meeting Bioelectromagn. Soc./Eur. BioElectromagn. Assoc., 2016, pp. 289-293.

[8] O. Bottauscio, M. Chiampi, and L. Zilberti, "A hybrid FE-BE method for SAR estimate in voxel based human models undergoing MRI," Eng. Anal. Boundary Elements, vol. 49, pp. 15-21, 2014.

[9] O. Bottauscio, M. Chiampi, L. Zilberti, and M. Zucca, "Evaluation of electromagnetic phenomena induced by transcranial magnetic stimulation," IEEE Trans. Magn., vol. 50, no. 2, pp. 1033-1036, Feb. 2014.

[10] M. Cvetković, D. Poljak, and J. Haueisen, "Analysis of transcranial magnetic stimulation based on the surface integral equation formulation," IEEE Trans., Biomed. Eng., vol. 62, no. 6, pp. 1535-1545, Jun. 2015.

[11] J. P. Reilly and A. Hirata, "Low-frequency electrical dosimetry: Research agenda of the IEEE International Committee on Electromagnetic Safety," Phys. Med. Biol., vol. 61, no. 12, pp. R138-R149, 2016.

[12] M. Stuchly and O. Gandhi, "Inter-laboratory comparison of numerical dosimetry for human exposure to $60 \mathrm{~Hz}$ electric and magnetic fields," Bioelectromagnetics, vol. 21, no. 3, pp. 167-174, 2000
[13] A. Hirata et al., "Intercomparison of induced fields in Japanese male model for ELF magnetic field exposures: Effect of different computational methods and codes," Radiation Protection Dosimetry, vol. 138, no. 3 , pp. 237-244, 2010.

[14] I. Laakso and A. Hirata, "Fast multigrid-based computation of the induced electric field for transcranial magnetic stimulation," Phys. Med. Biol., vol. 57, no. 23, 2012, Art. no. 7753.

[15] X.-L. Chen et al., "Analysis of human brain exposure to low-frequency magnetic fields: A numerical assessment of spatially averaged electric fields and exposure limits," Bioelectromagnetics, vol. 34, no. 5, pp. 375-384, 2013.

[16] D. Poljak, C. Y. Tham, O. Gandhi, and A. Sarolic, "Human equivalent antenna model for transient electromagnetic radiation exposure," IEEE Trans. Electromagn. Compat., vol. 45, no. 1, pp. 141-145, Feb. 2003.

[17] R. W. King, "A review of analytically determined electric fields and currents induced in the human body when exposed to $50-60-\mathrm{Hz}$ electromagnetic fields," IEEE Trans. Antennas Propag., vol. 52, no. 5, pp. 1186-1192, May 2004.

[18] C. M. Furse and O. Gandhi, "Calculation of electric fields and currents induced in a millimeter-resolution human model at $60 \mathrm{~Hz}$ using the FDTD method," Bioelectromagnetics, vol. 19, no. 5, pp. 293-299, 1998.

[19] T. W. Dawson, J. De Moerloose, and M. A. Stuchly, "Hybrid finitedifference method for high-resolution modelling of low-frequency electric induction in humans," J. Comput. Phys., vol. 136, no. 2, pp. 640-653, 1997.

[20] A. Chiba, K. Isaka, Y. Yokoi, M. Nagata, M. Kitagawa, and T. Matsuo, "Application of finite element method to analysis of induced current densities inside human model exposed to $60-\mathrm{Hz}$ electric field," IEEE Trans. Power App. Syst., vol. PAS-103, no. 7, pp. 1895-1902, Jul. 1984.

[21] A. Chiba and K. Isaka, "Analysis of current densities induced inside a human model by the two-step process method combining the surface-charge integral equation and the finite-element method," Electron. Commun. Japan (Part II: Electron.), vol. 79, no. 4, pp. 102-111, 1996.

[22] M. C. Gonzalez, A. Peratta, and D. Poljak, "Boundary element modeling of the realistic human body exposed to extremely-low-frequency (ELF) electric fields: Computational and geometrical aspects," IEEE Trans. Electromagn. Compat., vol. 49, no. 1, pp. 153-162, Feb. 2007.

[23] O. Bottauscio, M. Chiampi, and L. Zilberti, "Boundary element approaches for the evaluation of human exposure to low frequency electromagnetic fields," IEEE Trans. Magn., vol. 45, no. 3, pp. 1674-1677, Mar. 2009.

[24] K. H. Chan, J. Hattori, I. Laakso, A. Hirata, and M. Taki, "Computational dosimetry for grounded and ungrounded human models due to contact current," Phys. Med. Biol., vol. 58, no. 15, 2013, Art. no. 5153.

[25] V. De Santis, X. L. Chen, I. Laakso, and A. Hirata, "An equivalent skin conductivity model for low-frequency magnetic field dosimetry," Biomed. Phys. Eng. Exp., vol. 1, no. 1, 2015, Art. no. 015201.

[26] A. Hirata et al., "Intercomparison of induced fields in Japanese male model for ELF magnetic field exposures: effect of different computational methods and codes," Radiation Protection Dosimetry, vol. 138, no. 3, pp. 237-244, 2010.

[27] P. Dimbylow, "Development of the female voxel phantom, NAOMI, and its application to calculations of induced current densities and electric fields from applied low frequency magnetic and electric fields," Phys. Med. Biol., vol. 50, no. 6, 2005, Art. no. 1047.

[28] I. Laakso and A. Hirata, "Reducing the staircasing error in computational dosimetry of low-frequency electromagnetic fields," Phys. Med. Biol., vol. 57, no. 4, pp. N25-N34, 2012.

[29] E. Neufeld et al., "Computational platform combining detailed and precise functionalized anatomical phantoms with EM-Neuron interaction modeling," in Proc. XXXIth URSI Gen. Assembly Sci. Symp., 2014, pp. 1-4.

[30] J. Bakker et al., "Children and adults exposed to low-frequency magnetic fields at the ICNIRP reference levels: Theoretical assessment of the induced electric fields," Phys. Med. Biol., vol. 57, no. 7, 2012, Art. no. 1815.

[31] E. Neufeld, B. Lloyd, and N. Kuster, "From image-based modeling to the modeling of imaging with the Virtual Population," in Proc. Int. Workshop Simul. Synthesis Med. Imag., 2016, pp. 45-54.

[32] M. A. Garvey and V. Mall, "Transcranial magnetic stimulation in children," Clin. Neurophysiol., vol. 119, no. 5, pp. 973-984, May 2008.

[33] T. Rajapakse and A. Kirton, "Non-invasive brain stimulation in children: applications and future directions," Transl. Neurosci., vol. 4, no. 2, pp. 1-29, Jun. 2013.

[34] M. I. Iacono et al., "MIDA: A multimodal imaging-based detailed anatomical model of the human head and neck," PloS One, vol. 10, no. 4, 2015, Art. no. e0124126. 
[35] O. Bottauscio, M. Chiampi, and L. Zilberti, "Boundary element solution of electromagnetic and bioheat equations for the simulation of SAR and temperature increase in biological tissues," IEEE Trans. Magn., vol. 48, no. 2, pp. 691-694, Feb. 2012.

[36] O. Bottauscio, M. Chiampi, J. Hand, and L. Zilberti, "A GPU computational code for eddy-current problems in voxel-based anatomy," IEEE Trans. Magn., vol. 51, no. 3, pp. 1-4, Mar. 2015.

[37] I. Laakso, T. Uusitupa, and S. Ilvonen, "Comparison of SAR calculation algorithms for the finite-difference time-domain method," Phys. Med. Biol., vol. 55, no. 15, pp. N421-N431, 2010.

[38] W. C. Chew, M. S. Tong, and B. Hu, Integral Equation Methods for Electromagnetic and Elastic Waves. San Rafael, CA, USA: Morgan \& Claypool, 2009.

[39] T. Dawson, M. Potter, and M. Stuchly, "Evaluation of modeling accuracy of power frequency field interactions with the human body," Appl. Comput. Electromagn. Soc. J., vol. 16, no. 2, pp. 162-172, 2001.

[40] J. Massey et al., "AustinMan and AustinWoman: High fidelity reproducible and open-source electromagnetic voxel models," in Proc. 34th Annu. Meet. Bioelectromagnetics Soc., 2012, pp. 259-262.

[41] C. Warren, A. Giannopoulos, and I. Giannakis, "gprMax: Open source software to simulate electromagnetic wave propagation for ground penetrating radar," Comput. Phys. Commun., vol. 209, pp. 163-170, 2016.

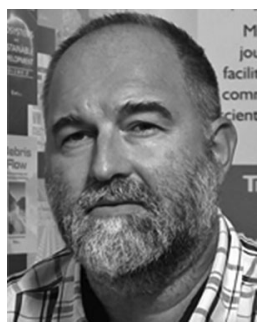

Dragan Poljak (SM'13) received the B.Sc. degree in electrical engineering in 1990, the M.Sc. degree in electrical engineering in 1994, and the Ph.D. degree in electrical engineering in 1996 from the University of Split, Split, Croatia.

He is a Full Professor with the Department of Electronics, Faculty of Electrical Engineering, Mechanical Engineering and Naval Architecture, University of Split. He is also an Adjunct Professor with Wessex Institute of Technology (WIT), U.K., and a member of WIT Board Directors. He was the Vice-Dean for research with the Faculty of Electrical Engineering, Mechanical Engineering, and Naval Architecture from 2011 to 2015. He became a member of WIT Board of Directors in 2011. His research interests include frequency- and time-domain computational methods in electromagnetics, particularly in the numerical modeling of thin wire configurations, human exposure to electromagnetic radiation, biomedical applications of electromagnetic fields, and magnetohydrodynamics.

Prof. Poljak became a member of the Board of the Croatian Science Foundation in June 2013. He is currently involved in three COST projects, ITER physics EUROfusion collaboration, and a Working Group 2 of the IEEE ICES TC95, SC6 "EMF Dosimetry Modeling."

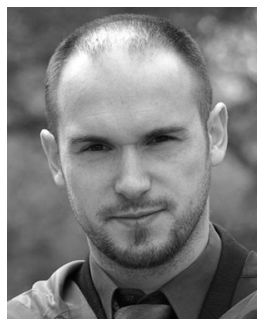

Mario Cvetković (M'09) was born in 1981. He received the B.S. degree in electrical engineering from the University of Split, Split, Croatia, in 2005, the M.Phil. degree in engineering and electrical engineering from Wessex Institute of Technology, University of Wales, Cardiff, U.K., in 2009, and the Ph.D. degree in engineering and electrical engineering, from the University of Split in December 2013.

He is currently a Postdoctoral Researcher with the Faculty of Electrical Engineering, Mechanical Engineering and Naval Architecture, University of Split. To date, he has authored or coauthored 38 journal and conference papers and two book chapters. His research interests include numerical modeling, computational bioelectromagnetics, and heat-transfer-related phenomena.

Dr. Cvetković received the Best Student Paper Award at the 16th edition SoftCOM. At the Scientific Novices Seminar held in 2012, he was awarded with the recognition for his previous scientific achievements. He is a member and a secretary of Working Group 2 (EMF Dosimetry Modeling) of IEEE/ICES TC95 SC6.

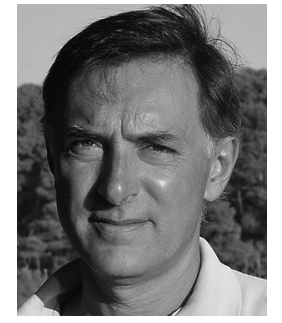

Oriano Bottauscio (SM'15) was born in 1961. He received the M.S. degree in electrical engineering from the Politecnico di Torino, Turin, Italy, in 1985.

$\mathrm{He}$ is the Research Director with the Istituto Nazionale di Ricerca Metrologica (INRIM), Turin, where he was responsible of the Applied Electromagnetics Department from 2001 to 2006 and has been the Head of the Division of Metrology for Quality of Life since 2015. He has authored or coauthored more than 200 scientific papers, with more than 2000 citations and h-index equal to 19 , and several national and international patents. From 1996 to 2001, he was a contract Professor in electrical engineering and a member of the Board of Professors of the Ph.D. School in Metrology, Politecnico di Torino, in convention with INRIM. His research activity is devoted to computational electromagnetism, with main reference to bioelectromagnetics, electromagnetic dosimetry related to medical equipment, electromagnetic fields and human exposure, and magnetic field mitigation.

Mr. Bottauscio was an Associate Editor (in 2009 and 2011) and Chief Editor (in 2013) of the special issues of the IEEE TRANSACTIONS ON MAGNETICS related to the Soft Magnetic Materials Conference. He was responsible and participate to several national and European research projects. From 2001 to 2005, he was a member of CENELEC TC 106X "Electromagnetic field in human environment"-WG3 "Measurement and calculation procedures in electric, magnetic and electromagnetic fields $(0 \mathrm{~Hz}-300 \mathrm{GHz})$ " and from 2002 to 2009 a member of the CIGRE Task Force C4.204 "Magnetic Field Mitigation Techniques." Since 2016, he has been a member of the IEEE TC95, SC6 "EMF Dosimetry Modeling," Working Group 2.

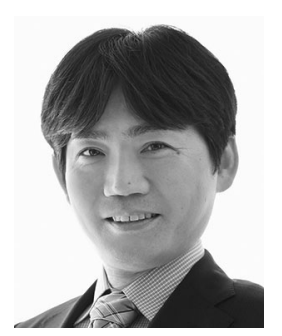

Akimasa Hirata (S'98-M'01-SM'10-F'17) received the B.E., M.E., and Ph.D. degrees in communications engineering from Osaka University, Suita, Japan, in 1996, 1998, and 2000, respectively.

He was a Research Fellow of the Japan Society for the Promotion of Science (JSPS Research Fellow) from 1999 to 2001, and also a Visiting Research Scientist with the University of Victoria, Canada, in 2000. In 2001, he joined the Department of Communications Engineering, Osaka University, as an Assistant Professor. In 2004, he joined the Department of Computer Science and Engineering, Nagoya Institute of Technology, Nagoya, Japan, as an Associate Professor and is currently a Full Professor. His research interests include electromagnetics and thermodynamics in biological tissue, waveguide analysis, EMC and EMI, and computational techniques in electromagnetics.

Dr. Hirata is a member of the Editorial Board of Physics in Medicine and Biology, a member of the main commission and a Chair of project group of the International Commission on Non-Ionizing Radiation Protection, and a member of Administrative Committee and a Subcommittee (EMF Dosimetry Modeling) Chair of the IEEE International Committee on Electromagnetic Safety, and an expert of World Health Organization. He was also an Associate Editor of the IEEE TRANSACTIONS ON BIOMEDICAL ENGINEERING from 2006 to 2012. He received several awards including Prizes for Science and Technology (Research Category 2011, Public Understanding Promotion Category 2014) by the Commendation for Science and Technology by the Minister of Education, Culture, Sports, Science and Technology, Japan, and IEEE EMC-S Technical Achievement Award. He is a Fellow of the Institute of Physics and a member of the Institute of Electronics, Information and Communication Engineers, the Institute of Electrical Engineers of Japan, and the Bioelectromagnetics Society. 


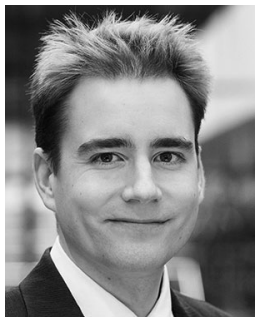

Ilkka Laakso (M'14) received the M.Sc.(Tech.) degree in electromagnetics from Helsinki University of Technology, Espoo, Finland, in 2007, and the D.Sc.(Tech.) degree in electromagnetics from Aalto University, Espoo, in 2011.

Between 2013 and 2015, he has been a Research Assistant Professor and Research Associate Professor with the Department of Computer Science and Engineering, Nagoya Institute of Technology. Since 2015, he has been an Assistant Professor in electromagnetics in health technologies with Aalto University. He has authored more than 75 papers published in international journals and conference proceedings. His research interests include computational bioelectromagnetic modeling for assessment of human safety and biomedical applications.

Dr. Laakso has received several awards, including the Student Award in International Symposium on EMC, Kyoto, 2009; the Ericsson Young Scientist Award, 2011; and the Young Scientist Award in URSI Commission B International Symposium on Electromagnetic Theory, Hiroshima, Japan, 2013. He is the Secretary of Subcommittee "EMF Dosimetry Modeling" of the IEEE International Committee on Electromagnetic Safety and a member of the scientific expert group of International Commission on Non-Ionizing Radiation.

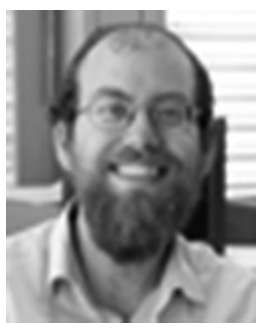

Esra Neufeld received the Dipl. Natw. (M.Sc.) degree in interdisciplinary sciences (main topics: theoretical physics, quantum electronics, organic and physical chemistry; master's thesis: computational sciences) and the Ph.D. degree in electrical engineering from the Computer Vision Laboratory, Swiss Federal Institute of Technology, Zürich, Switzerland, in 2004 and 2008, respectively.

Before that, he studied philosophy, theology, and law in the U.K. In 2004, he joined the Foundation for Research on Information Technologies in Society, Switzerland, where he has been the Head of the Computational Life Sciences Group since 2008. He is responsible for the development of a multiphysics simulation platform targeted at the life sciences as well as applied studies in this area and serves as a Consultant to companies and government agencies on MRI safety of implants and exposure safety, contributing to international standards. Since 2009, he has been employed partly as the Chief Scientific Officer at ZurichMedTech. He has authored and coauthored numerous journal and conference papers as well as book chapters and serves as a Scientific Reviewer for various journals and government agencies. His main research interests include the development of simulation and treatment planning software, personalized modeling, solver and algorithm development, medical image analysis and anatomical model generation, applied simulations particularly in the field bioelectromagnetics, interactions between physical exposure and physiology (e.g., EM- \& FUS-induced heating and neuromodulation), measurement technology, uncertainty assessment, and standardization.

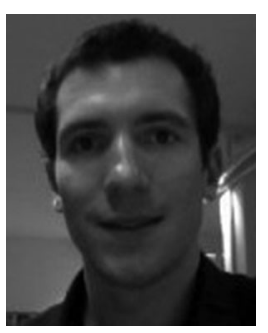

Sylvain Reboux was born in eastern France in 1982 He received the Engineering degree in mathematics and mechanics from the Ecole Polytechnique Paris, Paris, France, in 2005, the M.Sc. degree in fluid mechanics from the Université Pierre and Marie Curie, Paris, in 2005, and the Ph.D. degree in applied mathematics from the University of Nottingham, Nottingham, U.K., in 2008.

He worked for four years as a Postdoctoral Researcher with the Department of Computer Science, ETH Zürich (ETHZ), Zürich, Switzerland, developing novel computational methods and algorithms in a high-performance computing environment. He then joined ASCOMP AG, an ETHZ spinoff company specializing in computational fluid dynamics solutions. As the Head of research there, his role was to initiate and develop research projects in collaboration with universities and research institutes, as well as consultancy projects for industry. In August 2015, he joined ZMT Zurich MedTech, Zürich, as a Senior Software Engineer for computational life sciences.

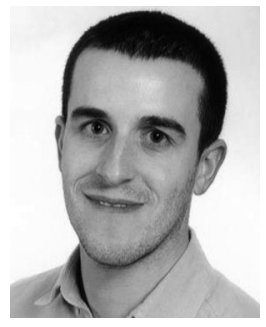

Craig Warren received the B.Eng. degree in electrical and mechanical engineering and the Ph.D. degree in engineering from the University of Edinburgh, Edinburgh, U.K., in 2003 and 2009, respectively.

From 2010 to 2017, he was a Teaching Fellow and Research Fellow with the University of Edinburgh. He is currently a Vice Chancellor's Senior Fellow in Future Engineering with Northumbria University, Newcastle upon Tyne, U.K. His main research interests include the development and application of nondestructive testing techniques for monitoring and investigation of critical infrastructure. He has a specific interest in the numerical modeling and optimization of electromagnetic sensing systems, such as a ground penetrating radar. $\mathrm{He}$ is also active in the fields of engineering education and technology enhanced learning.

Dr. Warren is a Chartered Engineer, a Fellow of the Higher Education Academy, U.K., and a member of both the Institution of Mechanical Engineers, U.K., and the Institution of Engineering Technology, U.K.

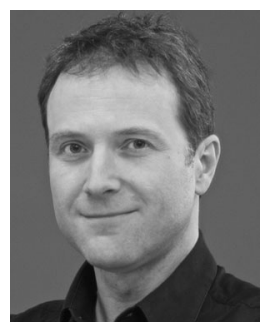

Antonis Giannopolous received the B.Sc. degree in geology from Aristotle University of Thessaloniki, Thessaloniki, Greece, in 1991, and the D.Phil. degree in electronics from the University of York, York, U.K., in 1997.

Since 2009, he has been a Senior Lecturer with the School of Engineering, Institute for Infrastructure and Environment, The University of Edinburgh, Edinburgh, U.K. His research interests include computational electromagnetics and in particular the application of the finite-difference time-domain (FDTD) method on the numerical modelling of ground penetrating radar. In addition, he works on the development and application of geophysical techniques for infrastructure sensing applications. He created gprMax, a freely available FDTD simulator, and he is directing its continuous development and enhancement. He was the General Chair of the 9th International Workshop on Advanced Ground Penetrating Radar, Edinburgh, 2017.

Dr. Giannopolous is a member of society of exploration geophysicists (SEG) and european association of geoscientists and Engineers (EAGE).

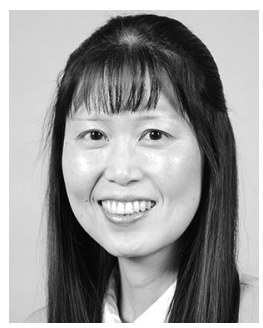

Fumie Costen (M'07-SM'12) received the B.Sc. and M.Sc. degrees in electrical engineering in 1991 and 1993, respectively, and the Ph.D. degree in informatics from Kyoto University, Kyoto, Japan.

From 1993 to 1997, she was with Advanced Telecommunication Research International, Kyoto, where she was involved in research on directionof-arrival estimation based on multiple signal classification algorithm for 3-D laser microvision. She was invited to give five talks in Sweden and Japan during 1996-2014. From 1998 to 2000, she was with Manchester Computing, University of Manchester, Manchester, U.K., where she was involved in research on metacomputing and has been a Lecturer since 2000. She filed three patents from the research in Japan in 1999. She filed a patent from the research on the boundary conditions in 2012 in the U.S. Her current research interests include computational electromagnetics in such topics as a variety of the finite-difference time-domain (FDTD) methods for microwave frequency range and high-spatial resolution, FDTD subgridding and boundary conditions, the hardware acceleration of the computation using general-purpose computing on graphics processing units, streaming single instruction multiple data extension, and advanced vector extension instructions.

Dr. Costen received the ATR Excellence in Research Award in 1996 and the Best Paper Award from the 8th International Conference on High-Performance Computing and the Networking Europe in 2000. 